Table DS2) show that coordinated treatment is typically lacking even in higher-income countries. Indeed, the median number of visits in the past 12 months among patients receiving treatment for mental disorders in general medical services is no different in high-income (1.5) than in low-/lower-middle-income (1.4) countries and only slightly higher in upper-middle-income countries (2.1). We also found that the proportion of patients prematurely terminating primary care treatment of mental disorders is quite high in high-income countries (35.4\%) as well as in lower-income countries (52.5\% for both groups).

Although Basu \& Arya consider the World Mental Health question on stopping treatment irrelevant to relationships with spiritual or religious healers, great care was taken in crafting the question sequence in which this question was embedded to be broadly applicable across treatment sectors and countries. The sequence began by asking respondents whether they ever in their life saw any of the professionals on a long country-specific customised list, for problems with their emotions, nerves, or use of alcohol or drugs. Respondents who reported having done so were asked whether they saw each type of professional for such problems in the past 12 months and, if so, number of visits, perceived helpfulness and whether or not they were still seeing the professional for these problems. Only those who said they had stopped seeing the professional were then asked, 'Did you complete the full recommended course of treatment? Or did you quit before the [provider] wanted you to stop?' I agree with Basu \& Arya that the framing of this question and of the response options may not have been the most natural way to describe an on-going relationship with a spiritual or religious healer, and I agree that customisation might well yield important new information. However, we would expect reports of having 'stopped' to be lower-bound estimates of the extent to which care for on-going emotional problems lacked continuity, so the high proportions of patients in lower-income countries who gave such reports are cause for deep concern. Basu \& Arya also note correctly that data on reasons for terminating treatment, including stigma, were not reported in the paper. Such data exist in the World Mental Health Surveys and will be presented in future reports.

Jessie E. Wells, Research Professor, University of Otago, Christchurch, New Zealand Email: elisabeth.wells@otago.ac.nz

doi: 10.1192/bjp.202.5.383b

\section{Psychological therapies in anorexia nervosa: on the wrong track?}

Recently, in a randomised controlled trial, specialist supportive clinical management (SSCM) has proven to be more effective than the Maudsley Model of Anorexia Nervosa Treatment for Adults (MANTRA), a treatment specially designed to address the disorder according to a rather complex rationale in comparison with SSCM. ${ }^{1}$ Specialist supportive clinical management, originally 'non-specific supportive clinical management' administered to a control group in a previous randomised controlled trial, ${ }^{2}$ was found to be more effective than two specialised treatments cognitive-behavioural therapy and interpersonal therapy - and was as effective as these treatments at 5-year follow-up. ${ }^{3}$

Specialist supportive clinical management was originally defined as clinical management and supportive psychotherapy, as revealed by its original definition:

'Non-specific supportive clinical management was developed for the present study, and its aim was to mimic outpatient treatment that could be offered to individuals with anorexia nervosa in usual clinical practice. It combined features of clinical management and supportive psychotherapy. Clinical management includes education, care, and support and fostering a therapeutic relationship that promotes adherence to treatment. Supportive psychotherapy aims to assist the patient through use of praise, reassurance and advice. The abnormal nutritional status and dietary patterns typical of anorexia nervosa were central to non-specific supportive clinical management, which emphasised the resumption of normal eating and the restoration of weight and provided information on weight maintenance strategies, energy requirements and relearning to eat normally. Information was provided verbally and as written handouts.' (p. 742)

In contrast, MANTRA claims to be novel in several respects: (a) it is biologically informed and trait-focused, drawing on neuropsychological, social cognitive and personality trait research; (b) it includes both intra- and interpersonal maintaining factors and strategies to address these; and (c) it is modularised with a hierarchy of procedures tailored to the individuals (as described in the authors' online Table DS1). ${ }^{1}$

Current treatment of anorexia nervosa is disheartening. Following successful weight restoration, almost $50 \%$ of patients relapse after 1-year follow-up, and pharmacological or psychological treatment persistently fails to neutralise the purported mechanisms underlying anorexia psychopathology. ${ }^{4}$ Against this backdrop, according to the American Psychological Association Task Force criteria for the Promotion and Dissemination of Psychological Procedures, SSCM could be the first treatment for adult anorexia to attain the consideration of a well-established psychosocial intervention. However, the acronym SSCM disguises the fact that it has entered the stage through the back door of non-specific supportive treatments originally assigned to control groups, and SSMC efficacy over advanced treatments that have a sound theoretical basis raises perplexing questions. Maybe we are on the wrong track by persistently failing to understand either the fundamental features articulating the current concept of the disorder in terms of symptoms, personality traits, psychopathology and neuropsychological profile, or that these features are an epiphenomenon of malnutrition and are thus irrelevant as targets for treatment. Rather than delving into the self, perhaps the focus should be on the starvation side of self-starvation. ${ }^{5}$

1 Schmidt U, Oldershaw A, Jichi F, Sternheim L, Startup H, McIntosh V, et al. Out-patient psychological therapies for adults with anorexia nervosa: randomised controlled trial. Br J Psychiatry 2012; 201: 392-9.

2 McIntosh VV, Jordan J, Carter FA, Luty SE, McKenzie JM, Bulik CM, et al Three psychotherapies for anorexia nervosa: a randomized, controlled trial. Am J Psychiatry 2005; 162: 741-7.

3 Carter FA, Jordan J, McIntosh VV, Luty SE, McKenzie JM, Frampton CM, et al. The long-term efficacy of three psychotherapies for anorexia nervosa: a randomized, controlled trial. Int J Eat Disord 2011; 44: 647-54.

4 Attia E, Walsh BT. Behavioral management for anorexia nervosa. $N$ Engl J Med 2009; 360: 500-6.

5 Gutierrez E. A rat in the labyrinth of anorexia nervosa: contributions of the activity-based anorexia rodent model to the understanding of anorexia nervosa. Int J Eat Disord 2013; doi: 10.1002/eat.22095. (Epub ahead of print.)

Emilio Gutierrez, Professor of Clinical Psychology, Universidad de Santiago de Compostela, Spain. Email: emilio.gutierrez@usc.es; Olaia Carrera, Universidad de Santiago de Compostela, Spain

doi: 10.1192/bjp.202.5.384

Authors' reply: We share Gutierrez \& Carrera's frustration about the difficulty in treating adults with anorexia nervosa. However, we disagree with their interpretation of our findings, and several other points they make.

First, in our trial specialist supportive clinical management (SSCM) was not superior to our new treatment, the Maudsley Model of Anorexia Treatment for Adults (MANTRA). In fact, outcomes for both interventions were similar. Moreover, in the 